\title{
Cement Mantle Thickness at the Bone Cement Interface in Total Knee Arthroplasty: Comparison of PS150 RP and LPS-Flex Knee Implants
}

Dong Oh Ko, MD, Song Lee, MD, Kyung Tae Kim, MD, Jae Il Lee, MD, Jin Woo Kim, MD, and Seong Min Yi, MD Department of Orthopedic Surgery, Seoul Sacred Heart General Hospital, Seoul, Korea

Purpose: To analyze the thickness of cement mantle at the bone cement interface in knees with closed and open box designs in total knee arthroplasty (TKA).

Materials and Methods: Eighty cases of TKA were performed from October 2013 to March 2014. The average age of the patients was 68.4 years. All patients were women and they were divided into two groups: group I with a closed box implant (PS150 RP, n=40) and group II with an open box implant (LPS-Flex, $\mathrm{n}=40$ ). We measured the cement mantle thickness at the bone cement interface from the distal femur and proximal tibia. If the thickness was $>1 \mathrm{~mm}$, it was considered an outlier.

Results: The mean cement mantle thickness at the interface was $1.4 \mathrm{~mm}$ in the distal femur and $0.8 \mathrm{~mm}$ in the proximal tibia. The value exceed $1 \mathrm{~mm}$ in 40 cases $(50 \%)$ in the distal femur and in 6 cases $(7.5 \%)$ in the proximal tibia $(p<0.001)$. The mean cement mantle thickness measured in the distal femur was $1.7 \mathrm{~mm}$ in group I and $1.0 \mathrm{~mm}$ in group II. The value exceed $1 \mathrm{~mm}$ in 32 cases (80\%) in group I and in 8 cases (20\%) in group II (p<0.000). Conclusions: The cement mantle at the interface was thicker in the knees with the closed box implant than those with the open box implant in TKA, especially in the distal femoral area. A thick cement mantle at the interface should be avoided because it affects the gap balance. In case of using a closed box implant in TKA, cementing should be performed with extra care.

Keywords: Knee, Arthroplasty, Thickness, Cement mantle

\section{Introduction}

The thickness of cement mantle has a profound influence on the stability of implant fixation in total knee arthroplasty (TKA). There are two types of bone cement mantles in the knee according to the location, one that is supposed to penetrate into the cancellous bone after TKA and the other that forms an interface between the implant and the bone ${ }^{1-6)}$.

Received February 15, 2016; Revised (1st) April 28, 2016;

(2nd) May 26, 2016; Accepted June 12, 2016

Correspondence to: Kyung Tae Kim, MD

Department of Orthopedic Surgery, Seoul Sacred Heart General

Hospital, 259 Wangsan-ro, Dongdaemoon-gu, Seoul 02488, Korea

Tel: +82-2-966-1616, Fax: +82-2-968-2394

E-mail: kktkimos@hanmail.net

This is an Open Access article distributed under the terms of the Creative Commons Attribution Non-Commercial License (http://creativecommons.org/licenses/by-nc/4.0/) which permits unrestricted non-commercial use, distribution, and reproduction in any medium, provided the original work is properly cited.
A thin cement mantle weakens the durability and fixation strength of a prosthesis implanted in the knee whereas a thick mantle increases the risk of thermal-induced osteonecrosis. The optimum thickness to allow for interdigitation of the cement into the cancellous bone has been suggested as $3-5 \mathrm{~mm}^{7-11}$. During cementing in TKA, it is also important to ensure impaction of the implant onto the bone because a thick cement mantle can reduce the gap width and bone-implant contact at the interface ${ }^{12}$. Knee implants are designed to have a $1 \mathrm{~mm}$ built-in cement pocket to maintain a proper mantle thickness after squeezing out of the cement by impaction ${ }^{13)}$ (Fig. 1). Therefore, the cement mantle at the bone cement interface has been recommended to be $\leq 1 \mathrm{~mm}$ in the proximal tibia and distal femur each to minimize its influence on the gap width, and $\geq 2 \mathrm{~mm}$ thickness should be avoided ${ }^{12}$.

Cementing techniques are essential to obtain a proper mantle thickness. Since cementing is affected by various factors including the type of cement, mixing time, temperature, and the surgeon's experience, it is important to utilize reproducible cementing 

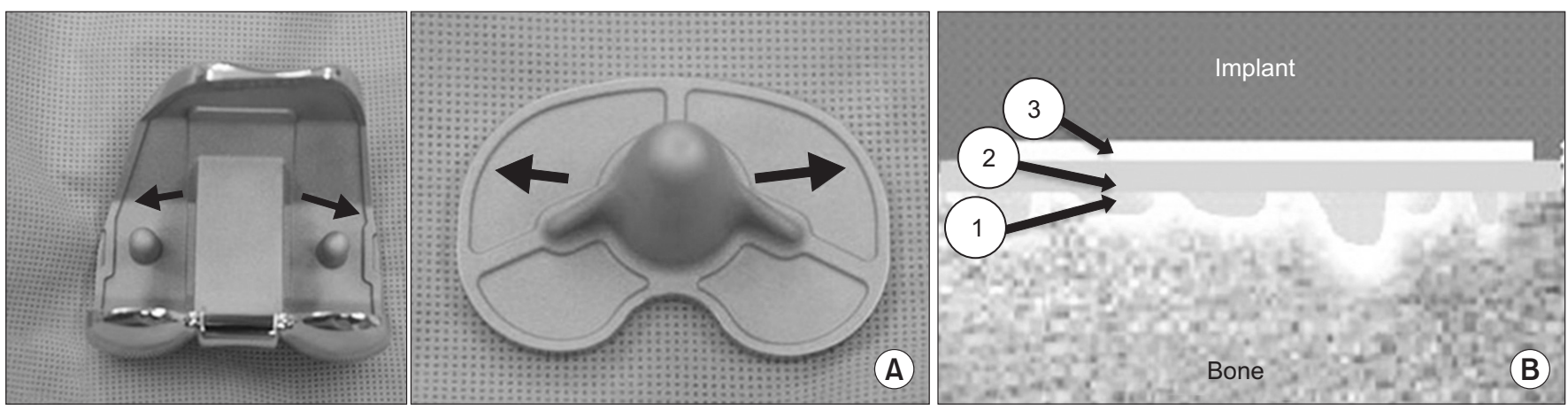

Fig. 1. (A) Cement pocket (arrow) for constant cement filling in the undersurface of an implant. (B) Composition of cement in the bony resection area: 1) cement infiltration into the cancellous bone; 2) cement mantle at the interface; and 3) cement mantle in the cement pocket.

techniques. We observed the cement mantle was thicker than the recommended thickness in some of our TKA patients and suspected the influence of the cement technique used and implant design. Therefore, we conducted this study to compare the difference with the targeted cement mantle thickness and to determine the influence of implant design based on the comparison of the open box design and the closed box design.

\section{Materials and Methods}

We enrolled 80 patients who underwent TKA for degenerative arthritis of the knee between October 2013 and March 2014 in this prospective, non-randomized study. The surgery was performed by the same surgeon using the same technique in all patients. The indication for surgery was degenerative knee arthritis. We included only female patients to rule out the influence of gender. Their mean age was 68.4 years. The exclusion criterion was severe bone defect that requires structural bone grafting or metal augmentation. Patients were divided into group I $(n=40)$ and group II ( $n=40)$ according to the type of implant used. Group I consisted of patients who received the closed box design (PFC Sigma PS150 RP; DePuy, Warsaw, IN, USA) whereas patients in group II received the open box design (Nexgen LPS-Flex; Zimmer, Warsaw, IN, USA).

There was no statistically significant difference between the groups in terms of baseline age, body mass index, bone mineral density, limitation in range of motion, and coronal plane deformity (Table 1).

\section{Surgical Technique}

The surgery was performed using the gap balancing technique $^{14)}$. Following resection of the distal femur and the proximal tibia along the mechanical axis, the coronal lower limb alignment
Table 1. Demographics

\begin{tabular}{lcc}
\hline \multicolumn{1}{c}{ Parameter } & Group I $(\mathrm{n}=40)$ & Group II $(\mathrm{n}=40)$ \\
\hline No. of knees & 40 & 40 \\
Sex (female) & 40 & 40 \\
Age $(\mathrm{yr})$ & $66.4 \pm 0.2$ & $66.1 \pm 0.1$ \\
Body mass index $\left(\mathrm{kg} / \mathrm{m}^{2}\right)$ & $25.3 \pm 0.5$ & $24.9 \pm 0.3$ \\
Bone mineral density $\left(\mathrm{g} / \mathrm{cm}^{2}\right)$ & $-1.9 \pm 0.1$ & $-1.8 \pm 0.2$ \\
Range of motion $\left(^{\circ}\right)$ & $128.2 \pm 0.6$ & $129.8 \pm 0.4$ \\
Varus deformity $\left(^{\circ}\right)$ & $9.5 \pm 0.3$ & $9.3 \pm 0.2$ \\
\hline
\end{tabular}

Values are presented as mean \pm standard deviation $(p>0.05)$.

and extension/flexion gap balance were assessed with a spacer block inserted. Soft tissue release was performed, if additional correction was considered necessary. With the knee in $90^{\circ}$ flexion, the rotational alignment of the femur in parallel to the resected tibial surface was determined using a ligament tensor and anteroposterior resection of the femur was performed. With a trial implant inserted, the lower extremity alignment, ligament balance, and gap balance in extension and flexion were assessed. The extension angle on the sagittal plane was determined to be $0^{\circ}-5^{\circ}$.

In all knees, the identical cementing technique was performed using a bag (40 g) of medium viscosity cement (Simplex P; Stryker, Mahwah, NJ, USA). Using a vacuum mixing bowl, polymer powder and monomer liquid were mixed for 1 minute until the liquid stage. At the doughy stage where the cement does not stick to a surgical glove, it was evenly applied to the bone surface and the implant including the box area. The bone surface was washed using a pulsatile lavage machine to remove residual blood or debris and dried with gauze. The implant was inserted in the order of tibial component, femoral component, and patellar component in one step. The cement was finger pressed to allow better 


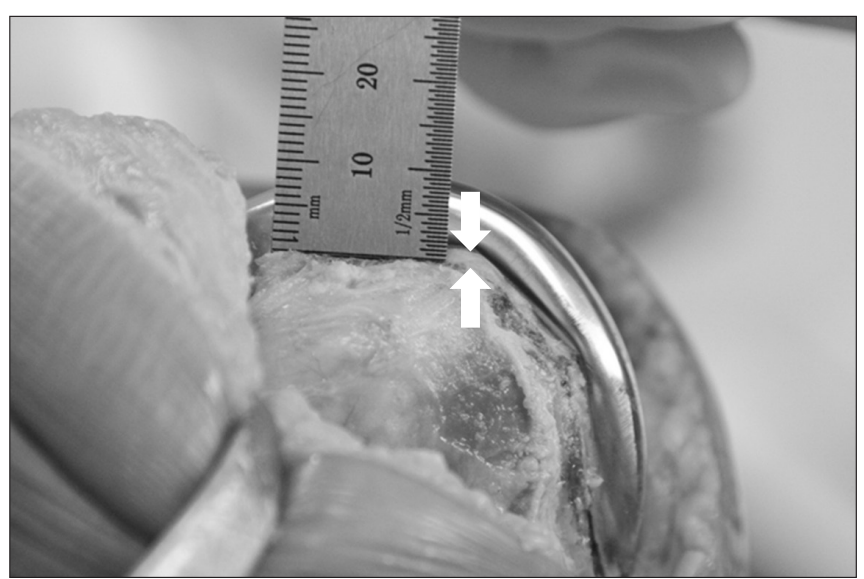

Fig. 2. Measurement of cement mantle thickness at the interface in the distal femur.

penetration into the cancellous bone. The implant was impacted into place onto the bone surface using an impactor. Any residual cement was removed while maintaining the extension alignment until the cement hardens.

\section{Assessment}

Both the open and closed box designs are not conducive to postoperative radiographic assessment of the cement mantle in the distal femur. Therefore, the mediolateral thickness of the cement at the interface was measured intraoperatively after implant insertion in the distal femur and proximal tibia using a $0.5 \mathrm{~mm}$ graduated ruler. To minimize measurement errors, two investigators performed measurements twice each and used the most accurate value for analysis (Fig. 2). An outlier was defined as 1) a mantle thickness of $>1 \mathrm{~mm}$ in the distal femur or proximal tibia or 2) a combined value of $>2 \mathrm{~mm}$. Statistical analysis was performed using the SPSS ver. 16.0 (SPSS Inc., Chicago, IL, USA). The Student $t$-test and chi-square test were used to compare the cement mantle thickness between the groups. A p-value of $\leq 0.05$ was considered statistically significant.

\section{Results}

The combined mantle thickness in the femur and tibia was $2.1 \pm 0.7 \mathrm{~mm}$ in 80 knees and the value was $>2 \mathrm{~mm}$ in 16 knees (20\%). The mean thickness in the distal femur was $1.3 \mathrm{~mm}$ in the medial area and $1.4 \mathrm{~mm}$ in the lateral area. The value in the proximal tibia was $0.8 \mathrm{~mm}$ and $0.8 \mathrm{~mm}$, respectively, showing no mediolateral difference $(\mathrm{p}>0.05)$. The mean cement thickness at the interface was $1.4 \mathrm{~mm}$ in the distal femur and $0.8 \mathrm{~mm}$ in the proximal tibia. The value was $\geq 1 \mathrm{~mm}$ in 40 knees (50\%)
Table 2. Comparison of Cement Mantle Thickness at the Interface between the Closed and Open Box Implants

\begin{tabular}{lccc}
\hline \multicolumn{1}{c}{ Parameter } & $\begin{array}{c}\text { Group I } \\
(\mathrm{n}=40) \\
\text { Closed box }\end{array}$ & $\begin{array}{c}\text { Group II } \\
(\mathrm{n}=40) \\
\text { Open box }\end{array}$ & p-value \\
\hline Cement mantle thickness & & & \\
Total $(\mathrm{mm})$ & $2.6 \pm 0.6$ & $1.6 \pm 0.5$ & 0.000 \\
Femoral $(\mathrm{mm})$ & $1.7 \pm 0.4$ & $1.0 \pm 0.3$ & 0.000 \\
Tibial $(\mathrm{mm})$ & $0.9 \pm 0.3$ & $0.6 \pm 0.2$ & 0.000 \\
Total $>2 \mathrm{~mm}(\%)$ & $14(35)$ & $2(5)$ & 0.002 \\
Femoral $>1 \mathrm{~mm}(\%)$ & $32(80)$ & $8(20)$ & 0.000 \\
Tibial $>1 \mathrm{~mm}(\%)$ & $6(15)$ & $0(0)$ & 0.034 \\
\hline
\end{tabular}

Values are presented as mean \pm standard deviation or case.

in the distal femur, which was high compared to 6 knees $(7.5 \%)$ in the proximal tibia $(\mathrm{p}<0.001)$. The cement thickness was significantly greater in group I patients with the closed box design $(2.6 \pm 0.6 \mathrm{~mm})$ than group II patients with the open box design $(1.6 \pm 0.5 \mathrm{~mm})(\mathrm{p}<0.001)$. The combined value was $>2 \mathrm{~mm}$ in 14 knees (35\%) in group I and in II knees (5\%) in group II, showing statistically significant intergroup difference $(\mathrm{p}<0.01)$. The value was greater in both the distal femur and proximal tibia in group I $(1.7 \pm 0.4 \mathrm{~mm}$ and $0.9 \pm 0.3 \mathrm{~mm}$, respectively) than group II (1.0 \pm 0.3 $\mathrm{mm}$ and $0.6 \pm 0.2 \mathrm{~mm}$, respectively) $(\mathrm{p}<0.001)$. The incidence of $>1 \mathrm{~mm}$ thickness in the distal femur and proximal tibia was significantly higher in group I (32 [80\%] and $6[15 \%]$, respectively) than group II (8 [20\%] and $0[0 \%]$, respectively) $(\mathrm{p}<0.05)$ (Table 2$)$.

\section{Discussion}

In this study, we compared the thickness of the cement mantle in the distal femur and proximal tibia with the preoperatively planned target thickness in our TKA patients who had implants with two different box designs. The cement mantle thickness in the distal femur was thicker in the knees with the closed box design than in those with the open box design. Therefore, our findings suggest that particular care should be taken when using a closed box design in TKA.

Stable implant fixation for prevention of component loosening can be obtained with the use of cement that penetrates into the cancellous bone or forms a mantle at the interface between the bone and the implant. Cement penetration into the pore openings of the cancellous bone (interdigitation) facilitates bone to cement bonding and prevents ingress of micro-particulate debris into the tibia, obviating the risk of component loosening and osteolysis ${ }^{5,7,8,15}$. Although cement is strong under compression, it 

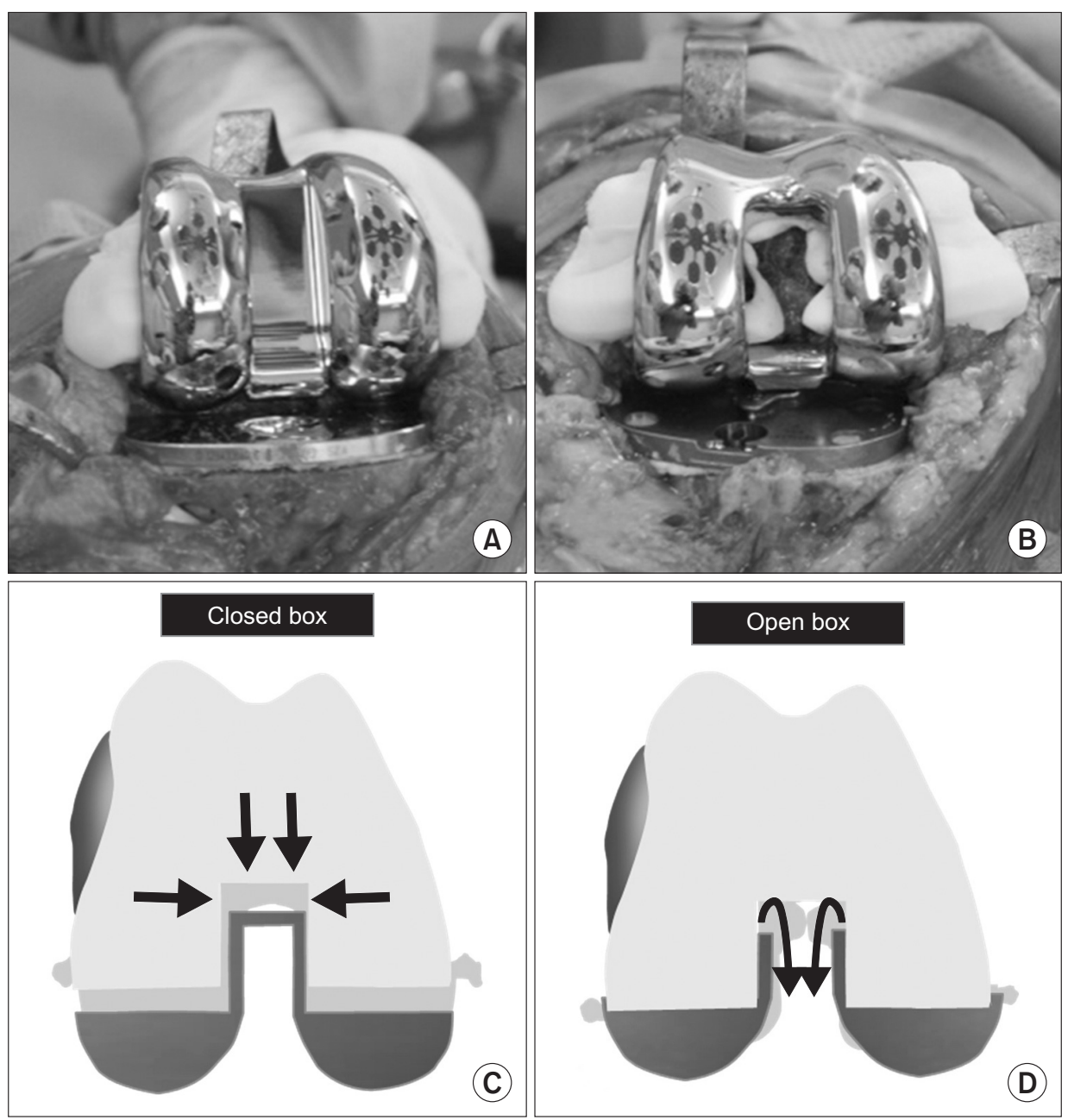

Fig. 3. (A, B) Cement leakage from the periphery of the closed box type implant and from the periphery and central box of the open box type implant. (C) Less cement leakage in the box area (arrows) can prevent compression of the femoral component in the case of closed box type implant. (D) Cement can easily leak (arrows) and the implant can be compressed in the case of open box type implant. has a low shear strength. Therefore, to obtain an optimal cement bone interface shear strength through interdigitation, a 3-5 mm cement penetration depth has been advised. Techniques using a spatula, finger pressing, and a cement gun have been suggested to improve penetration of cement into bone $\mathrm{e}^{3,16,17)}$. In contrast, the ideal thickness of cement mantle that bonds the prosthesis to the bone at the interface has been considered $\leq 1 \mathrm{~mm}^{12}$. Due to the presence of a $1 \mathrm{~mm}$ cement pocket in the implant, the mantle thickness can be maintained after impaction. Therefore, considering its impact on the gap kinematics, the thickness should be $\leq 1 \mathrm{~mm}$ in the distal femur and proximal tibial each. However, in our patients, the mean mantle thickness was $\geq 1 \mathrm{~mm}$ in the distal femur (mean, $1.3 \mathrm{~mm}$ ) although it was less than $<1 \mathrm{~mm}$ in the proximal tibia (mean, 0.8 ). In addition, the prevalence of $\geq 1 \mathrm{~mm}$ thickness in the femur was $50 \%$ whereas it was only $7.5 \%$ in the tibia.

Factors that can influence the cement mantle thickness include cementing techniques and implant designs. Cementing tech- niques consist of the cement mixing process and implant fixation process. The former process refers to polymerization of methyl methacrylate (polymethyl methacrylate) by mixing polymer powder and monomer liquid. According to changes in morphology of the cement, it can be divided into 4 stages (wetting stage, liquid stage, doughy stage, curing stage, and setting stage ${ }^{12,18)}$ ). In the doughy stage, the cement no longer adheres to a surgical glove so that it can be applied to an implant and the bone, and the implant should be inserted into the knee in this stage. The length of doughy stage is variable ranging from 3 to 7 minutes according to the type of cement, storage method, temperature, and mixing speed. Proper fixation of the femoral and tibial components within the optimal application time frame is critically dependent on the surgeon's experience ${ }^{19,20)}$. We mixed the polymer powder and monomer liquid for 1 full minute and started to apply it to the implant and the cut surface when it was relatively thin to secure sufficient time for cementing. Bone cements can be divided into three types (low, medium, and high) according to viscosity. 
The higher the viscosity is, the shorter the interval between mixing and doughy stage, which affects the cementing technique. In TKA, high or medium viscosity cement is commonly used ${ }^{5,21,22)}$. For proper implant fixation, the components should be impacted into position onto the bone surface. During this procedure, excess cement that leaks between the implant and the bone surface should be removed. Then, to ensure the absence of excess cement at the interface, the component is pressed with an impactor or with an insertion of a trial bearing in knee extension under axial loading until the cement is cured.

Although we used the same cementing technique in all patients, the cement mantle thickness was larger in some patients. We think this can be attributed to the implant design. TKA can be performed either with an open box femoral component or a closed box femoral component. The open box design has a cam in the box area revealing the bone. It requires an additional time for thorough cement removal to prevent contact of residual cement with the bearing post; however, it necessitates small bone resections and allows for intramedullary Kirschner wire insertion in case of periprosthetic fracture. In the closed box design, the box is covered by metal acting as a cam exhibiting the opposite advantages and disadvantages of the open box design ${ }^{12}$. We compared the cement mantle thickness between the two designs under the hypothesis that the closed box design would disrupt leaking of the excess cement between the prosthesis and the bone surface resulting in a thicker cement mantle. The combined cement mantle thickness in the distal femur and the proximal tibia was larger in the closed box design group than in the open box design group ( $2.6 \pm 0.6 \mathrm{~mm}$ vs. $1.6 \pm 0.5 \mathrm{~mm})$. The percentage of knees with a value of $>2 \mathrm{~mm}$ was also higher in the closed box design group than in the open box design group ( $35 \%$ vs. $5 \%$ ). In particular, the value in the distal femur was higher in the closed box design group than in the open box design group (1.7 \pm 0.4 $\mathrm{mm}$ vs. $1.0 \pm 0.3 \mathrm{~mm}$ ) and $>1 \mathrm{~mm}$ was significantly more prevalent in the closed box design group than in the open box design group ( $80 \%$ vs. $20 \%$ ). All these findings appear to corroborate our hypothesis. It is our understanding that the closed box design provides a broader contact with the bone surface than the open box design and reduces postoperative bleeding, but the excess cement contained in the box even after impaction disrupts stable fixation of the prosthesis to the bone surface ${ }^{6}$ (Fig. 3). Since there was no significant difference in the tibial component design, we did not expect the cement mantle thickness in the tibia would be different between the groups. However, the incidence of $>1 \mathrm{~mm}$ thickness in the tibia was higher in the closed box design group than in the open box design group (15\% vs. $0 \%$ ). The open box
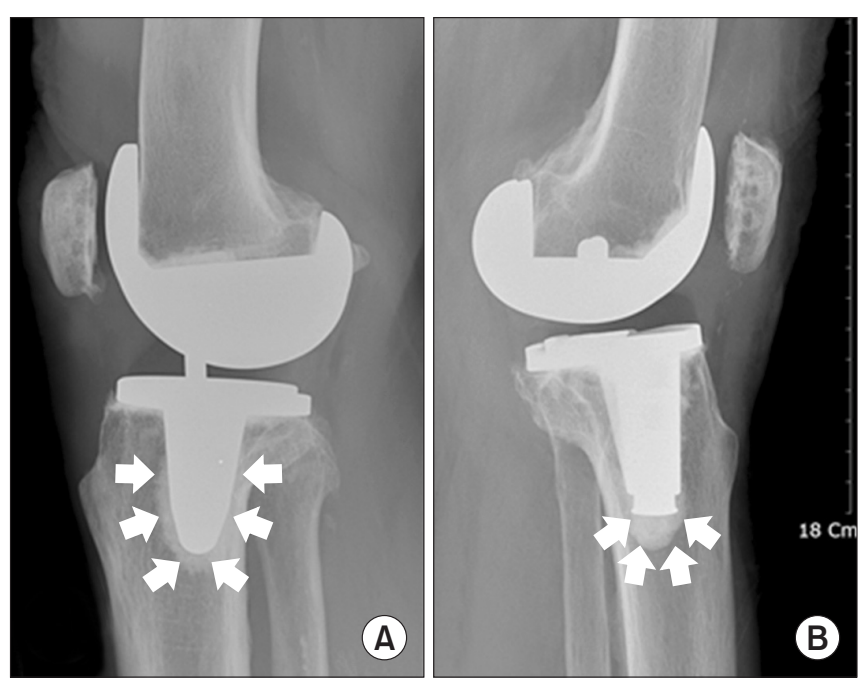

Fig. 4. (A) Cement filling (arrows) around the tibial stem of PS150 RP (closed box type) implant suggesting restriction of cement spread. (B) Deeper cement penetration into the medullary canal of tibia in LPS-Flex (open box type) implant.

design requires wide and deep tibial reaming of the medullary cavity whereas the closed box design allows for tapered reaming for the stem to better fit into the cavity, resulting in increased resistance during press-fit fixation of the tibial component. We surmise that the greater resistance of the closed box design had an influence on the cement thickness between the implant and the bone surface (Fig. 4).

One of the limitations of this study is that we included only one implant for each design in the analysis. Therefore, it is questionable whether our findings can be applied to other implants of each design, and our results should be confirmed by further research for generalization. In addition, we did not examine various factors that can influence the cement mantle thickness other than the cementing techniques and implant designs. Although the results could be affected by the extension gap tightness variance associated with the use of a $2 \mathrm{~mm}$ bearing insert and errors in bone resection and thickness measurement, we did not take these factors into consideration because the surgery was performed by the same surgeon using the same technique. Last, we did not investigate the clinical significance of the cement mantle thickness; however, it was beyond the scope of this study where the impact of implant design on cement mantle thickness in TKA was examined. We believe clinical outcomes should also be addressed in a future study based on a further follow-up.

We identified that the closed box design knee implant was associated with the greater cement mantle thickness than the open box design knee implant after TKA performed using the same 
surgical technique. Therefore, it is advised to take extra caution in cementing of the closed box design in TKA. In our opinion, it could be helpful to insert an implant when the cement is in a thinner stage or to use less amount of cement.

\section{Conclusions}

The cement mantle thickness was greater in the distal femur than in the proximal tibia in our TKA patients ( $1.4 \mathrm{~mm}$ vs. 0.8 $\mathrm{mm}$ ). In particular, the closed box design (PS150 RP) was associated with the greater cement mantle thickness than the open box design (LPS-Flex). We think that the closed box design obstructs leakage of residual cement, adversely affecting impaction of the implant. Therefore, extra care should be taken to avoid creating a thick cement mantle that affects the gap balance especially when a closed box design is used in TKA.

\section{Conflict of Interest}

No potential conflict of interest relevant to this article was reported.

\section{References}

1. Kopec M, Milbrandt JC, Duellman T, Mangan D, Allan DG. Effect of hand packing versus cement gun pressurization on cement mantle in total knee arthroplasty. Can J Surg. 2009;52:490-4.

2. Lutz MJ, Pincus PF, Whitehouse SL, Halliday BR. The effect of cement gun and cement syringe use on the tibial cement mantle in total knee arthroplasty. J Arthroplasty. 2009;24:461-7.

3. Schlegel UJ, Bishop NE, Püschel K, Morlock MM, Nagel K. Comparison of different cement application techniques for tibial component fixation in TKA. Int Orthop. 2015;39:4754.

4. Vanlommel J, Luyckx JP, Labey L, Innocenti B, De Corte R, Bellemans J. Cementing the tibial component in total knee arthroplasty: which technique is the best? J Arthroplasty. 2011;26:492-6.

5. Kopec M, Milbrandt JC, Kohut N, Kern B, Allan DG. Effect of bone cement viscosity and set time on mantle area in total knee arthroplasty. Am J Orthop (Belle Mead NJ). 2009;38:519-22.

6. Saari T, Li MG, Wood D, Nivbrant B. Comparison of cementing techniques of the tibial component in total knee replacement. Int Orthop. 2009;33:1239-42.

7. Norton MR, Eyres KS. Irrigation and suction technique to ensure reliable cement penetration for total knee arthroplasty. J Arthroplasty. 2000;15:468-74.

8. Walker PS, Soudry M, Ewald FC, McVickar H. Control of cement penetration in total knee arthroplasty. Clin Orthop Relat Res. 1984;(185):155-64.

9. Dorr LD, Lindberg JP, Claude-Faugere M, Malluche HH. Factors influencing the intrusion of methylmethacrylate into human tibiae. Clin Orthop Relat Res. 1984;(183):147-52.

10. Kim YH, Walker PS, Deland JT. A cement impactor for uniform cement penetration in the upper tibia. Clin Orthop Relat Res. 1984;(182):206-10.

11. Fukushima H, Hashimoto Y, Yoshiya S, Kurosaka M, Matsuda M, Kawamura S, Iwatsubo T. Conduction analysis of cement interface temperature in total knee arthroplasty. Kobe J Med Sci. 2002;48:63-72.

12. Cho WS, Kim MY, Youm YS. Knee joint arthroplasty. 3rd ed. Seoul: Young Chang; 2015. p48-51, 184.

13. Vertullo CJ, Davey JR. The effect of a tibial baseplate undersurface peripheral lip on cement penetration in total knee arthroplasty. J Arthroplasty. 2001;16:487-92.

14. Dennis DA, Komistek RD, Kim RH, Sharma A. Gap balancing versus measured resection technique for total knee arthroplasty. Clin Orthop Relat Res. 2010;468:102-7.

15. Mann KA, Ayers DC, Werner FW, Nicoletta RJ, Fortino MD. Tensile strength of the cement-bone interface depends on the amount of bone interdigitated with PMMA cement. J Biomech. 1997;30:339-46.

16. Cawley DT, Kelly N, McGarry JP, Shannon FJ. Cementing techniques for the tibial component in primary total knee replacement. Bone Joint J. 2013;95:295-300.

17. Vaninbroukx M, Labey L, Innocenti B, Bellemans J. Cementing the femoral component in total knee arthroplasty: which technique is the best? Knee. 2009;16:265-8.

18. Cameron HU, Mills RH, Jackson RW, Macnab I. The structure of polymethylmethacrylate cement. Clin Orthop Relat Res. 1974;(100):287-91.

19. Haas SS, Brauer GM, Dickson G. A characterization of polymethylmethacrylate bone cement. J Bone Joint Surg Am. 1975;57:380-91.

20. Krause WR, Krug W, Miller J. Strength of the cement-bone interface. Clin Orthop Relat Res. 1982;(163):290-9.

21. Silverman EJ, Landy DC, Massel DH, Kaimrajh DN, Latta LL, Robinson RP. The effect of viscosity on cement penetration in total knee arthroplasty, an application of the squeeze 
film effect. J Arthroplasty. 2014;29:2039-42.

22. Jiang J, Lv H, Zhu J. Influence of closed-box and open-box knee prosthesis on postoperative blood loss in patients un- dergoing total knee replacement. Zhongguo Xiu Fu Chong Jian Wai Ke Za Zhi. 2008;22:9-11. 